\title{
NONORIENTABLE SLICE GENUS CAN BE ARBITRARILY LARGE
}

\author{
Joshua Batson
}

\begin{abstract}
A generic cross-section of a surface smoothly embedded in four-space is a knot or a link. Every knot can be realized as the cross-section of some surface, but if that surface is required to be orientable, then work of Fox, Milnor, and Murasugi show that its genus may need to be quite large. For example, the $(2, n)$ torus knot is not a cross-section of any orientable surface with genus less than $n-1$, but is the cross-section of a Klein bottle. We give a lower bound on the first Betti number of a surface with cross-section a knot $K$ in terms of the signature of $K$ and the Heegaard-Floer $d$-invariant of the integer homology sphere given by -1 -surgery on $K$. In particular, we show that any smooth surface in four-space with cross-section the $(2 k, 2 k-1)$ torus knot has first Betti number at least $2 k-2$.
\end{abstract}

\section{Introduction}

Let $F$ be a surface smoothly embedded in $\mathbb{R}^{4}$. Viewing the first coordinate of $\mathbb{R}^{4}$ as time makes $F$ into a movie ${ }^{1}$ in which almost every frame is a link in $\mathbb{R}^{3}$. Figure 1 shows an embedded Klein bottle with the torus knot $T_{2,5}$ and the unknot as cross-sections.

Every knot $K \subset \mathbb{R}^{3}$ can be realized as a cross-section of some surface in four-space. For example, one may take two Seifert surfaces for $K$ placed in $\{ \pm 1\} \times \mathbb{R}^{3}$ together with the cylinder $[-1,1] \times K$; smooth the corners to get an embedded $F \subset \mathbb{R}^{4}$ with $K=F \cap\{0\} \times \mathbb{R}^{3}$. (All maps and manifolds we discuss will be smooth.) However, most knots cannot be realized as a cross-section of an embedded sphere - Milnor and Fox [1] showed that the Alexander polynomial of such a "slice" knot must factor as $\Delta_{K}(t)= \pm p(t) p\left(t^{-1}\right)$ for some polynomial $p$ with integer coefficients.

If we require the surface to be orientable, then some knots never appear as crosssections of surfaces with low genus. This was first shown by Murasugi, who proved that if $K$ is a cross-sectional slice of an orientable surface $F$, then the genus of $F$ is at least the absolute value of the knot signature $\sigma(K)$ [7]. The signature of $T_{2, n}$ is $-(n-1)$, for example, so any orientable surface with $T_{2, n}$ as a cross-section must have genus at least $n-1$.

Techniques from gauge theory, Floer homology, and Khovanov homology have given many additional obstructions to realizing certain knots as slices of low-genus orientable surfaces $[5,11,13]$. Since these bounds are tight for $T_{2, n}$, which is a slice of a Klein bottle, they do not apply to nonorientable surfaces. The global property of orientability, perhaps recast as the existence of a top homology class, a complex structure, or an infinite cyclic branched covering space, is used in a crucial way. Some obstructions

Received by the editors April 4, 2013.

${ }^{1}$ Ayumu Inoue has rendered an excellent movie of a sphere with cross-section the stevedore knot (http://www.youtube.com/watch?v=61IM9p6XOKo). 


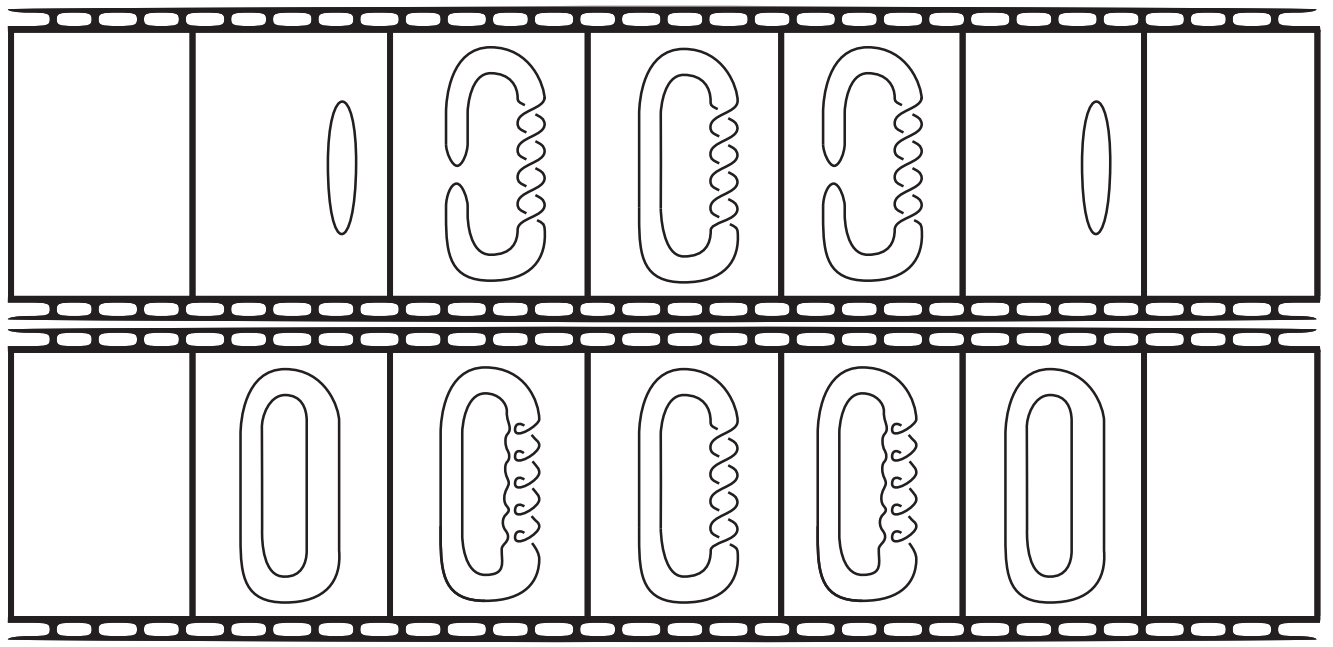

Figure 1. A Klein bottle and a genus four orientable surface in four-space, each with cross-section $T_{2,5}$. The topology of each surface can be deduced from its Euler characteristic (count births, deaths, saddles) and orientability (try to consistently orient the crosssections)

have been found to particular knots appearing as slices of a Klein bottle $\mathrm{Kl}$ or $\mathrm{Kl} \# \mathrm{Kl}$ in $\mathbb{R}^{4}$ ( $[6,16]$, see [3] especially for a comprehensive survey). But the possibility remained that every knot could be realized as a cross-section of, say, $\#^{3} \mathrm{Kl}$. We show that this is not the case.

Theorem 1.1. If the torus knot $T_{2 k, 2 k-1}$ is a cross-section of a smoothly embedded surface $F \subset \mathbb{R}^{4}$, then $b_{1}(F) \geq 2 k-2$.

Before explaining the lower bound, we make a few simplifications. Suppose that a knot $K$ is the intersection of a surface $F \subset \mathbb{R}^{4}$ with a hyperplane. That hyperplane cuts $F$ into two pieces $F_{1}$ and $F_{2}$, each of which has boundary $K$. If we add the point at infinity to $\mathbb{R}^{4}$ to form the four-sphere, then the knot $K$ lives in an "equatorial" $S^{3}$ and each $F_{i}$ lives in a "hemisphere" $B^{4}$. Doubling either half $\left(B^{4}, F_{i}\right)$ across the boundary will produce a new closed surface with cross-section $K$. Since $b_{1}(F)=$ $b_{1}\left(F_{1}\right)+b_{1}\left(F_{2}\right) \geq 2 \min \left(b_{1}\left(F_{i}\right)\right)$, a surface of minimal $b_{1}$ with cross-section $K$ can always be found by doubling. (Both surfaces in Figure 1 are doubles, as the movies are symmetric.) So we focus on understanding the following:

Definition 1.2. The (non)orientable slice genus of a knot $K \subset S^{3}$ is the smallest first Betti number of any smoothly embedded (non)orientable surface $F \subset B^{4}$ with boundary $K$.

To show that a knot is not the boundary of a surface with small first Betti number, we must bound both its orientable and nonorientable slice genus. We give a new bound for the latter. 
Theorem 1.3. Suppose that $K \subset S^{3}$ bounds a smoothly embedded, nonorientable surface $F \subset B^{4}$. Then

$$
b_{1}(F) \geq \frac{\sigma(K)}{2}-d\left(S_{-1}^{3}(K)\right)
$$

where $\sigma$ denotes the Murasugi signature and $d$ the Heegaard-Floer d-invariant of the integer homology sphere given by -1 surgery on $K$.

The strategy of the proof is as follows. Following Yasuhara, we first replace our nonorientable surface in $B^{4}$ with an orientable surface in another manifold:

Proposition 1.4 (Yasuhara [16]). Let $F \subset B^{4}$ be a smoothly embedded nonorientable surface with odd $b_{1}$ and boundary a knot $K \subset S^{3}$. Then there exists a smoothly embedded orientable surface $F^{\prime} \subset S^{2} \times S^{2} \backslash B^{4}$, also with boundary $K$, and with $b_{1}\left(F^{\prime}\right)=b_{1}(F)-1$ and $e\left(F^{\prime}\right)=e(F)+2$.

The expression $e(F)$ denotes the (relative) normal Euler number of $F$, a measure of the twisting of the normal bundle of $F$. (While orientable surfaces in $B^{4}$ always have trivial normal bundle, nonorientable ones never do.)

We then attach a -1-framed 2-handle along $K$ to get a four-manifold $\bar{W}$ with boundary $S_{-1}^{3}(K)$. There is a closed, orientable surface $\Sigma$ in $\bar{W}$ formed by the union of $F^{\prime}$ and the core of the 2-handle (See Figure 2). Excising a neighborhood of $\Sigma$ from $\bar{W}$ produces a negative semi-definite cobordism from a circle bundle over $\Sigma$ to $S_{-1}^{3}(K)$. The definiteness of $W$ gives us an inequality between the Heegaard-Floer $d$-invariants of its two boundaries, ultimately yielding:

Theorem 1.5. Suppose that $K \subset S^{3}$ bounds a smoothly embedded, nonorientable surface $F \subset B^{4}$. Then

$$
b_{1}(F) \geq \frac{e(F)}{2}-2 d\left(S_{-1}^{3}(K)\right) .
$$

To prove Theorem 1.3, we cancel the Euler number using:

Theorem 1.6 (Gordon and Litherland [4]). Suppose that $K \subset S^{3}$ bounds a smoothly embedded, nonorientable surface $F \subset B^{4}$. Then

$$
b_{1}(F) \geq \sigma(K)-\frac{e(F)}{2} .
$$

Computing the $d$-invariants in Theorem 1.3 is difficult in general, though there is an algorithm beginning with the filtered Heegaard Floer knot complex $C F K^{\infty}(K)$ [12]. When $K$ admits a lens space surgery, however, these $d$-invariants can be read off from the Alexander polynomial of $K$. Using a recursive formula of Murasugi for the signatures of torus knots, we can compute our lower bound for all torus knots.

There is a simple construction of nonorientable cobordisms between torus knots: pinch parallel strands, then pull taut. Beginning with $T_{p, q}$ and composing these cobordisms produces a surface $F_{p, q} \subset B^{4}$. (See Section 5.)

Conjecture 1.7. Suppose $T_{p, q}$ bounds a smoothly embedded surface $F \subset B^{4}$. Then $b_{1}(F) \geq b_{1}\left(F_{p, q}\right)$.

We show that this conjecture holds for infinitely many torus knots, including $T_{2 k, 2 k-1}$. The nonorientable slice genus of $T_{2 k, 2 k-1}$ is $k-1$. 

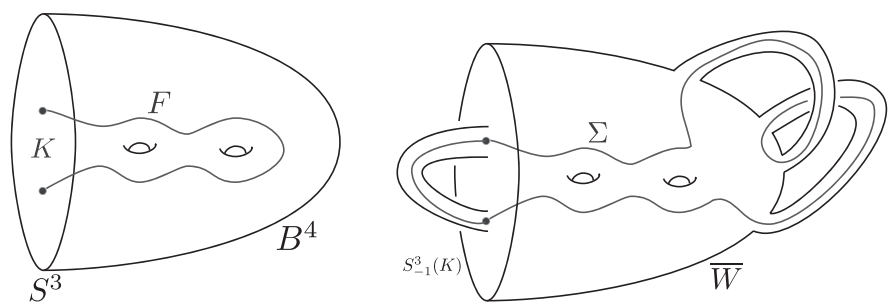

FiguRE 2. The proof in a picture. Start with a nonorientable surface $F$ in $B^{4}$ and construct with an orientable surface $\Sigma$ in $\bar{W}$.

It is interesting to compare with the Milnor conjecture, which states that a minimal genus orientable surface in $B^{4}$ with boundary $T_{p, q}$ can be isotoped into $S^{3}$. There is a Möbius band in $B^{4}$ with boundary $T_{4,3}$, but a punctured Klein bottle is best possible in $S^{3}[15]$.

Remark 1.8. Gessel et al. [2] have recently computed the $d$-invariants of circle bundles over nonorientable surfaces. This can be used to prove Theorem 1.5 without having to construct the orientable replacement.

\section{The signature inequality}

One of the major differences between orientable surfaces and nonorientable surfaces in four-space is that the latter can have nontrivial normal bundles. The normal bundle of a closed surface $F \subset B^{4}$ is determined by its (twisted) Euler number. This can be computed as a geometric self-intersection: take a transverse pushoff of $F$, and choose arbitrary orientations in the neighborhood of each intersection point. Together with the orientation of $\mathbb{R}^{4}$, this allows us to assign signs to each intersection; the sum, $e(F)$, turns out to be independent of the choice of pushoff and local orientation. If $F$ is orientable, then it represents an integral homology class and self-intersection can be computed as a cup product; since $H_{2}\left(B^{4} ; \mathbb{Z}\right)$ vanishes $e(F)$ must be zero. Even if $F$ is nonorientable, it still represents a homology class over over $\mathbb{Z} / 2$. Hence $e(F)$ must be even, although it need not be zero. For example, the $\mathbb{R P}^{2}$ in Figure 5 has normal Euler number 2.

If $F$ has boundary a knot $K \subset S^{3}$, then it has a relative normal Euler number. One way to compute $e(F)$ is to take an orientable Seifert surface $\Sigma$ for $K$ and find the normal Euler number of the closed surface $\Sigma \cup_{K} F$. Alternatively, one may take a nonvanishing section $s$ of the normal bundle $\nu(F)$ (one exists since $F$ retracts to its 1 -skeleton). The restriction of $s$ to the boundary provides a framing of $K$, and

$$
e(F)=-\operatorname{lk}(K, s(K)) \text {. }
$$

For example, the obvious Möbius band with boundary $T_{2, n}$ has normal Euler number $-2 n$.

We now sketch of the proof of the signature inequality Theorem 1.6. Let $F \subset B^{4}$ be a surface with boundary $K \subset S^{3}$. Let $W(F)$ denote the double cover of $B^{4}$ branched over $F$. Gordon and Litherland [4] use the $G$-signature theorem to show that the quantity

$$
\sigma(K):=\sigma(W(F))+\frac{e(F)}{2}
$$


is independent of the choice of surface $F$ with boundary $K$. For any such $F$ then,

$$
\left|\sigma(K)-\frac{e(F)}{2}\right|=|\sigma(W(F))| \leq b_{2}(W(F))=b_{1}(F) .
$$

This inequality is tight for the two natural surfaces with boundary $T_{2, n}$, which has signature $-(n-1)$. The Seifert surface has $e(F)=0$ and $b_{1}(F)=n-1$, while the Möbius band has $e(F)=-2 n$ and $b_{1}(F)=1$. The surfaces in Figure 1 can be constructed by taking the Möbius band or Seifert surface in $S^{3}$, pushing it into the four-ball, then doubling across the boundary.

In light of the important role played by $e(F)$, it may be clarifying to sort surfaces based on the framing they induce on the knot and try to minimize $b_{1}$ separately in each class. The signature inequality can be interpreted as stating that $2 \sigma(K)$ is a "preferred framing" for the knot $K$, deviation from which requires a more complex surface.

\section{Constructing an orientable replacement}

In this section, we prove

Proposition 1.4. Let $F \subset B^{4}$ be a smoothly embedded nonorientable surface with odd $b_{1}$ with boundary a knot $K \subset S^{3}$. Then there exists an orientable surface $F^{\prime} \subset$ $S^{2} \times S^{2} \backslash B^{4}$ which still bounds $K$, and has $b_{1}\left(F^{\prime}\right)=b_{1}(F)-1$ and $e\left(F^{\prime}\right)=e(F)+2$.

Proof. We break the proof into four steps.

Step 1: There is an embedded disk $D \subset B^{4}$, with boundary contained in $F$, such that $F \backslash \partial D$ is orientable.

Since $F$ has odd $b_{1}$, it is diffeomorphic to a punctured orientable surface boundaryconnect summed with a Möbius band (Figure 3). Let $\gamma \subset F$ be the core of the Möbius band, so that $F-\gamma$ is orientable. (The class $[\gamma]$ is Poincaré dual to $w_{1}$.)

After an ambient isotopy, we may arrange that $\gamma$ lies in a small sphere $S_{\epsilon}^{3}=\partial D_{\epsilon}^{4}$, where $D_{\epsilon}^{4}$ is a small disk at the center of $B^{4}$. Think of $\gamma$ as a knot, with some projection. Changing a few crossings will convert $\gamma$ to the unknot $U$, and this can be realized as an isotopy in $B^{4}$ pushing some strands a little outside of $D_{\epsilon}^{4}$, over, then back down. The unknot bounds an embedded disk in $S_{3}^{\epsilon}$, which we push in toward the center of $B^{4}$; that disk together with the trace of the isotopy is our embedded $D$. After a generic perturbation, $D$ will intersect $F$ transversely on its interior.

Let $N$ be a small regular neighborhood of $D$.

Step 2: The intersection $\partial N \cap F$ is a link $L \subset S^{3}$ of the form shown in Figure 3.

$N$ is diffeomorphic to $D \times D^{2}$, and intersects our surface $F$ in a Möbius band (in the neighborhood of $\partial D=\gamma$ ) and a collection of disks pt $\times D^{2}$ (neighborhoods of the transverse intersections of $F$ with the interior of $D$ ). If we draw $S^{3}=\partial N$ with its standard decomposition into solid tori $S^{3} \cong S^{1} \times D^{2} \cup_{T^{2}} D^{2} \times S^{1}$, we see $F \cap \partial N$ as the link $L$ consisting of a $(2,2 k+1)$-cable of the core of the first factor together with a collection of $l$ longitudes for the second. (Orient $S^{3}$ as the boundary of $N$.) The number of twists is determined by the Euler number: $2 k+1=-\frac{e(F)}{2}$.

By construction, $L$ bounds the disjoint union of a Möbius band and $l$ disks in $N$.

Step 3: $L$ bounds $l+1$ disjoint embedded disks in $S^{2} \times S^{2} \backslash B^{4}$ 


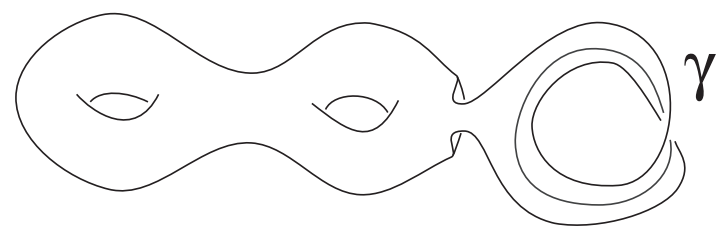

Figure 3 . The surface $F$.

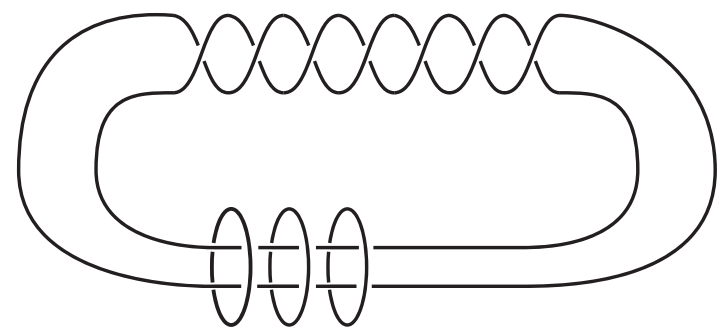

FiguRE 4. Our surface $F$ intersects $\partial N$ in a torus knot and an unlink.

Suzuki [14] has shown that every link is slice in $S^{2} \times S^{2} \backslash B^{4}$. Since we need control over the Euler numbers, we construct the slice disks explicitly.

A handle decomposition for $S^{2} \times S^{2} \backslash B^{4}$ consists of two zero-framed 2-handles $H_{1}$ and $H_{2}$ attached along a Hopf link in the boundary $S^{3}$, together with a 4 -handle. To construct the slice disks for $L$, we begin with $|k|+l$ parallel copies of the core of $\mathrm{H}_{2}$ and two parallel copies of the core of $H_{1}$-their boundaries form a "multi-Hopf" link, with components $U_{1}, \ldots, U_{|k|+l}, L_{1}, L_{2}$, as in the first frame of Figure 4 . For each $1 \leq i \leq|k|$, connect $U_{i}$ to $L_{1}$ with a twisted strip, and with one additional twisted strip, connect $L_{1}$ to $L_{2}$. Call the surface consisting of the parallel cores and the strips $E$, and note that the boundary of $E$ is isotopic to $L$. Since each strip connects a distinct disk to $L_{1}$, we see that $E$ is a collection of $l+1$ disjoint embedded disks with boundary $L$.

Step 4: Construct $F^{\prime}$, and compute $b_{1}\left(F^{\prime}\right)$ and $e\left(F^{\prime}\right)$.

If we excise $N$ from $B^{4}$, we are left with an orientable surface $F^{\prime \prime} \subset S^{3} \times[0,1]$, with boundary $K$ in $S^{3} \times\{0\}$ and $L$ in $S^{3} \times\{1\}$. Attach $S^{2} \times S^{2} \backslash B^{4}$ along $S^{3} \times\{1\}$ to form a larger manifold, still diffeomorphic to $S^{2} \times S^{2} \backslash B^{4}$. The slice disks $E$ for $L$ combine with $F^{\prime \prime}$ to form an orientable surface $F^{\prime}$, whose only remaining boundary is the original knot $K$.

Since we have removed $l$ disks and a Möbius band from $F$, and replaced them with $l+1$ disks, $b_{1}\left(F^{\prime}\right)=b_{1}(F)-1$. It remains to compare the normal Euler numbers. Since $F^{\prime \prime}$ is orientable and $H_{2}\left(S^{3} \times[0,1]\right)$ vanishes, $F^{\prime \prime}$ has trivial relative normal bundle. Thus $e(F)=e(F \cap N)$ and $e\left(F^{\prime}\right)=e(E)$. The disks in $F \cap N$ with boundary the unknots $U_{1}, \ldots, U_{l}$ have trivial self-intersection as well, since they can be added back into $F$ while maintaining orientability. The disks in $E$ with boundary the unknots are attached with framing 0 , so they also contribute nothing to the Euler number. The nontrivial contributions come from the Möbius band in $F \cap N$ with boundary the torus knot component of $L$ and the interesting disk in $E$. We invite the reader to 


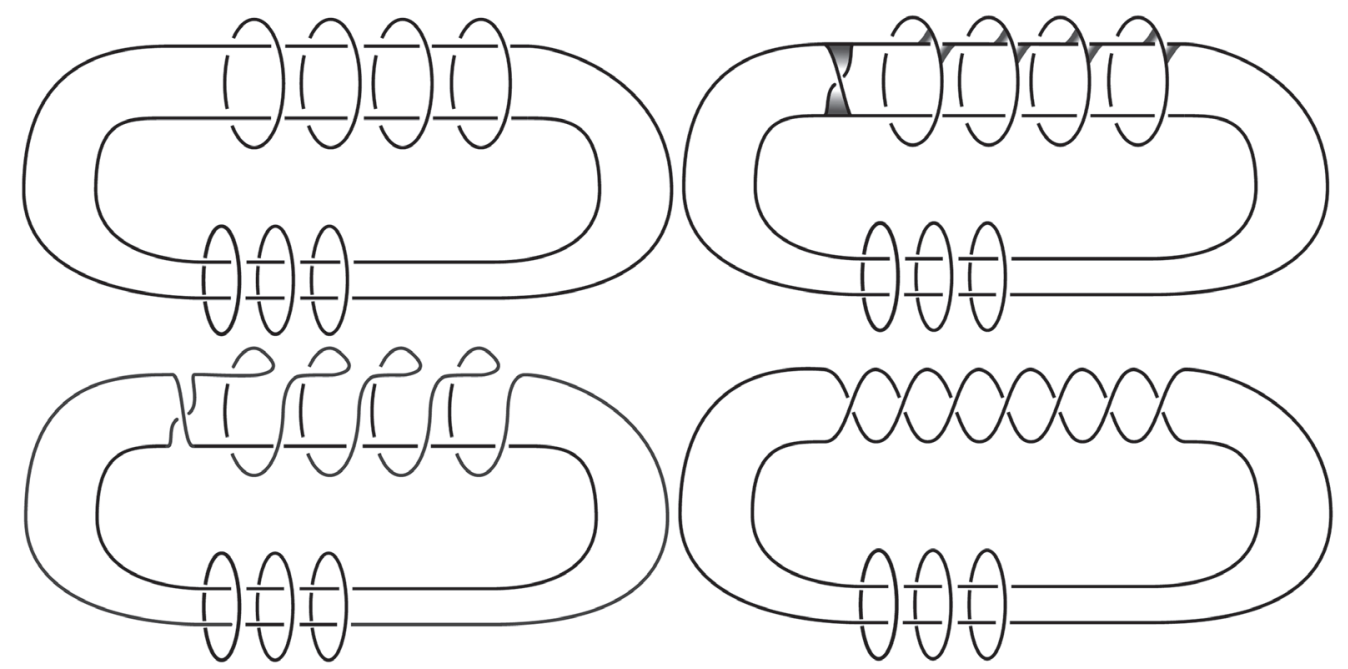

Figure 5. For $k=-4, l=3$, we have drawn the "multi-Hopf" link bounding a collection of parallel disks, the strips which join them to form $E$, and the boundary of $E$, which is isotopic to $L$.

verify that the induced framings differ by 2 , due to the difference between the vertical twisted strip connecting $V_{1}$ to $V_{2}$ in $E$ and the horizontal one in Möbius band. That is,

$$
e\left(F^{\prime}\right)=e(E)=e(F \cap N)+2=e(F)+2 .
$$

For future reference, we note that the homology class $\left[F^{\prime}\right] \in H_{2}\left(S^{2} \times S^{2} \backslash B^{4}\right)$ is $(2, m)$, in the basis given by $H_{1}$ and $H_{2}$. Since $F^{\prime}$ is orientable, its algebraic selfintersection number, $4 m$, computes its Euler number $e\left(F^{\prime}\right)$. Comparing with the formula for the number of twists in step 2 , we see that $m$ is just $-k$.

\section{4. $d$-invariants}

Heegaard Floer homology associates to a 3-manifold $Y$ equipped with a $\operatorname{Spin}^{c}$ structure $\mathfrak{t}$ a suite of $\mathbb{Z}[U]$-modules which fit into a long exact sequence:

$$
\cdots \rightarrow H F^{-}(Y, \mathfrak{t}) \stackrel{i}{\rightarrow} H F^{\infty}(Y, \mathfrak{t}) \stackrel{\pi}{\rightarrow} H F^{+}(Y, \mathfrak{t}) \stackrel{\delta}{\rightarrow} H F^{-}(Y, \mathfrak{t}) \rightarrow \cdots
$$

If $c_{1}(\mathfrak{t})$ is torsion (in which case we also say that $\mathfrak{t}$ is torsion), then there is a $\mathbb{Q}$ grading gr on the each of these groups which is respected by $i$ and $\pi$. The action of $U$ decreases grading by 2 . If $Y$ is a rational homology sphere, then every $\operatorname{Spin}^{c}$ structure $\mathfrak{t}$ is torsion, and $H F^{\infty}(Y, \mathfrak{t}) \cong \mathbb{Z}\left[U, U^{-1}\right]$. In that case, the $d$-invariant $d(Y, \mathfrak{t})$ is the minimal grading of a non- $\mathbb{Z}$-torsion element of $\mathrm{HF}^{+}(Y, \mathfrak{t})$ in the image of $\pi$.

If $b_{1}(Y)>0$, then there is an additional action of $H:=H_{1}(Y) /$ Tors on the $H F^{\circ}$ groups, which decreases grading by 1. If for every torsion $\mathfrak{t} \in \operatorname{Spin}^{c}(Y)$, we have $H F^{\infty}(Y, \mathfrak{t}) \cong \mathbb{Z}\left[U, U^{-1}\right] \otimes_{\mathbb{Z}} \Lambda^{*} H$, then we say that $Y$ has standard $H F^{\infty}$. In that case, there are many correction terms, one for each generator of $\Lambda^{*} H$. We will be concerned with the bottom-most correction term, $d_{b}(Y, \mathfrak{t})$, defined to be the minimal 


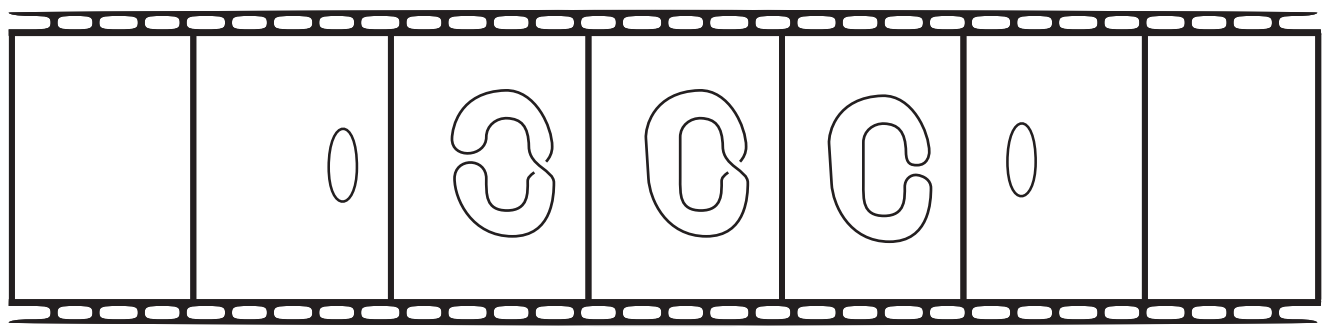

FiguRE 6. An embedded $\mathbb{R P}^{2}$ with normal Euler number 2.

grading of a nontorsion element of $H^{+}(Y, \mathfrak{t})$ in the image the restriction of $\pi$ to the kernel of the $H$-action on $H F^{\infty}(Y, \mathfrak{t})$. The $d$-invariants terms will be useful to us because of their relationship to definite cobordisms.

Proposition 4.1 ( [9]). Let $Y$ be a closed oriented 3-manifold (not necessarily connected) with standard $H F^{\infty}$, endowed with a torsion Spin ${ }^{c}$ structure $\mathfrak{t}$. If $X$ is a negative semi-definite four-manifold with boundary $Y$ such that the restriction map $H^{1}(X ; \mathbb{Z}) \rightarrow H^{1}(Y ; \mathbb{Z})$ is trivial, and $\mathfrak{s}$ is a Spin ${ }^{c}$ structure on $X$ restricting to $\mathfrak{t}$ on $Y$, then

$$
c_{1}(\mathfrak{s})^{2}+b_{2}^{-}(X) \leq 4 d_{b}(Y, \mathfrak{t})+2 b_{1}(Y) .
$$

We are now ready to prove our main inequality.

Theorem 1.5. Suppose that $K \subset S^{3}$ bounds a smoothly embedded, nonorientable surface $F \subset B^{4}$. Then

$$
b_{1}(F) \geq \frac{e(F)}{2}-2 d\left(S_{-1}^{3}(K)\right) .
$$

Proof. Both sides of the inequality change by the same amount under a positive real "blow-up." More precisely, if we connect sum $F \subset B^{4}$ with the embedding of $\mathbb{R P}^{2} \subset S^{4}$ with Euler number +2 pictured in Figure 5, then both $b_{1}$ and $e / 2$ increase by 1 . After blowing up sufficiently many times, we may assume that $e(F)>0$ and $b_{1}(F)$ is odd.

Using Proposition 1.4, we construct an orientable surface $F^{\prime} \subset S^{2} \times S^{2} \backslash B^{4}$ with boundary $K \subset S^{3}$. Attach a -1-framed 2-handle along $K$ to form a 4-manifold $\bar{W}$ with boundary $S_{-1}^{3}(K)$ and intersection form

$$
Q_{\bar{W}}=\left(\begin{array}{ccc}
-1 & 0 & 0 \\
0 & 0 & 1 \\
0 & 1 & 0
\end{array}\right) .
$$

We may cap off $F^{\prime}$ with the core of the 2-handle to form a closed surface $\Sigma$ with genus $g=\left(b_{1}(F)-1\right) / 2$, homology class $(1,2, m)$, and self-intersection

$$
n:=4 m-1=e(F)+1>0 .
$$

If we decompose $\bar{W}=\nu(\Sigma) \cup W$, then $W$ will be a negative semi-definite cobordism from $Y_{g, n}$, the Euler number $n$ circle bundle over $\Sigma$, to $S_{-1}^{3}(K)$. Alternatively, we can view $W$ as a negative semi-definite four-manifold with disconnected boundary $Y_{g,-n} \amalg S_{-1}^{3}(K)$. We need to understand the homology, $H F^{\infty}$, and $d$-invariants of $Y_{g, n}$ and $S_{-1}^{3}(K)$, and the intersection form on $W$. 

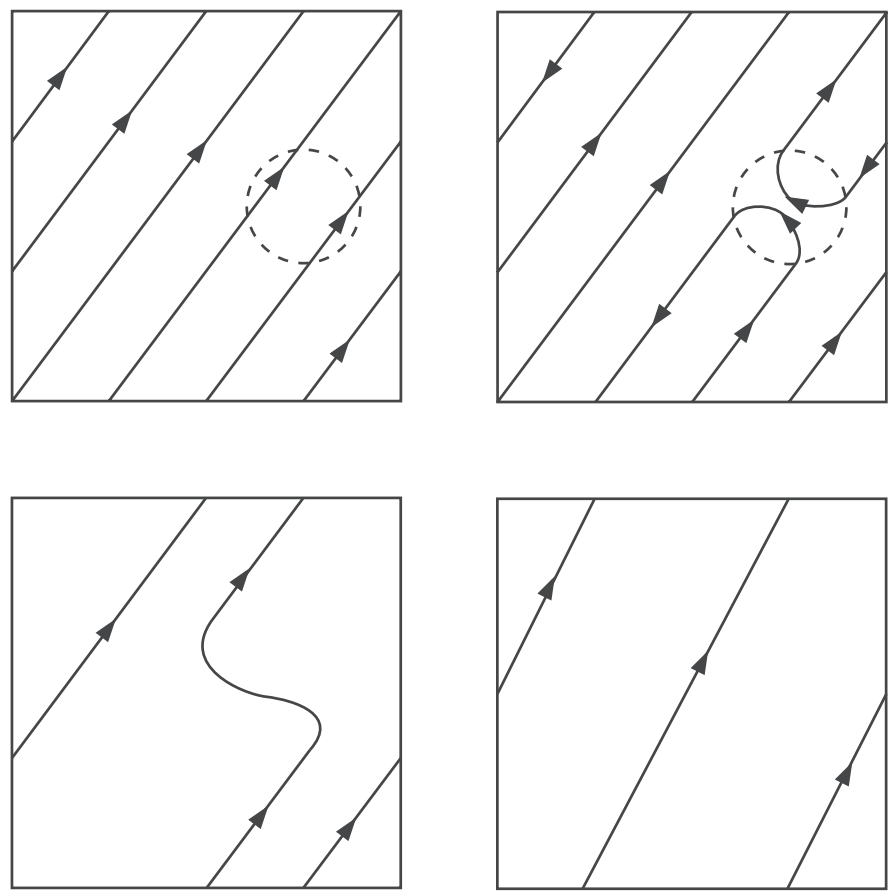

Figure 7. A cobordism from $T_{4,3}$ to $T_{2,1}$.

The Gysin sequence for the disk bundle $\nu(\Sigma)$ gives

$$
\begin{aligned}
& 0 \rightarrow H^{1}(\nu(\Sigma)) \rightarrow H^{1}\left(Y_{g, n}\right) \rightarrow H^{2}\left(\nu(\Sigma), Y_{g, n}\right) \\
& \stackrel{e}{\longrightarrow} H^{2}(\nu(\Sigma)) \rightarrow H^{2}\left(Y_{g, n}\right) \rightarrow H^{1}(\Sigma) \rightarrow 0
\end{aligned}
$$

where $e \in H^{2}(\nu(\Sigma)) \cong \mathbb{Z}$ is $n$ times the generator. Thus $H^{2}\left(Y_{g, n}\right) \cong \mathbb{Z}^{2 g} \oplus \mathbb{Z} / n$. Note that the restriction of $H^{1}(\nu(\Sigma))$ to $H^{1}\left(Y_{g, n}\right)$ is an isomorphism. Since $H^{1}(\bar{W})=0$ (no 1-handles were used in its construction), the Mayer-Vietoris sequence

$$
\begin{aligned}
0 \rightarrow H^{1}(\bar{W}) & \rightarrow H^{1}(\nu(\Sigma)) \oplus H^{1}(W) \rightarrow H^{1}\left(Y_{g, n}\right) \\
& \rightarrow H^{2}(\bar{W}) \rightarrow H^{2}(\nu(\Sigma)) \oplus H^{2}(W) \rightarrow H^{2}\left(Y_{g, n}\right)
\end{aligned}
$$

shows that $H^{1}(W)=0$, trivially satisfying the restriction hypothesis of Proposition 4.1. Since $H^{2}(\bar{W}) \cong \mathbb{Z}^{3}$ has no 2-torsion, a $\operatorname{Spin}^{c}$ structure on $\bar{W}$ is determined by its first Chern class. Any $\operatorname{Spin}^{c}$ structure on $W$ will give us some inequality between $d$-invariants, but we will only need to consider a certain $\operatorname{Spin}^{c}$ structure $\mathfrak{s}_{t}$ with $P D\left(c_{1}\left(\mathfrak{s}_{t}\right)\right)=( \pm 1,2,2 a)$, where

$$
a=\frac{2(m-g)-1 \pm 1}{4}
$$

and the sign is chosen so as to make $a$ an integer. (In the following \pm represents that same sign choice.) The given vector is characteristic for $Q_{\bar{W}}$, so does correspond to a $\operatorname{Spin}^{c}$ structure. Crucially for our later use, $c_{1}\left(\mathfrak{s}_{t}\right)$ evaluates to $n-2 g$ on $\Sigma$.

To compute the $c_{1}^{2}$ term in the proposition, we decompose the intersection form of $\bar{W}$ in terms of the $\mathbb{Q}$-valued cohomology intersection forms on $\nu(\Sigma)$ and $W$ : if 
$c \in H^{2}(\bar{W})$, then

$$
Q_{W}(c)=Q_{\nu(\Sigma)}\left(\left.c\right|_{\nu(\Sigma)}\right)+Q_{W}\left(\left.c\right|_{W}\right)
$$

A generator of $H^{2}\left(\nu(\Sigma), Y_{g, n}\right)$ maps to $n$ times the generator of $H^{2}(\nu(\Sigma))$ in the Gysin sequence above, so $Q_{\nu(\Sigma)}=\left(\frac{1}{n}\right)$. The value of $\left.c\right|_{\nu(\Sigma)} \in H^{2}(\nu(\Sigma))$ is determined by integrating it over $\Sigma$, giving

$$
Q_{\bar{W}}(c)=\frac{\langle c,[\Sigma]\rangle^{2}}{n}+Q_{W}\left(\left.c\right|_{W}\right)
$$

In our case,

$$
c_{1}\left(\left.\mathfrak{s}_{t}\right|_{W}\right)^{2}=Q_{\bar{W}}\left(c_{1}\left(\mathfrak{s}_{t}\right)\right)-\frac{\left\langle c_{1}\left(\mathfrak{s}_{t}\right),[\Sigma]\right\rangle^{2}}{n}=-1+8 a-\frac{(n-2 g)^{2}}{n}=-2 \pm 2-\frac{4 g^{2}}{n} .
$$

The relevant $d$-invariant of $Y_{g,-n}$ is computed in Section 9 of [9], for use in their proof of the Thom conjecture. If $n>2 g$, then

$$
d_{b}\left(Y_{g,-n},\left.\mathfrak{s}_{t}\right|_{Y_{g,-n}}\right)=\frac{1}{4}-\frac{g^{2}}{n}-\frac{n}{4} .
$$

That calculation uses the integer surgeries exact sequence associated to the Borromean knot in $K \subset \#^{2 g} S^{1} \times S^{2}$ : the $-n$ surgery on $K$ gives $Y_{g,-n}$. Since $\#^{2 g} S^{1} \times S^{2}$ has standard $H F^{\infty}$, so does $Y_{g,-n}$ (cf. Proposition 9.4 of [9]). Finally, since $S_{-1}^{3}(K)$ is an integer homology sphere, it also has standard $H F^{\infty}$.

We may now apply Proposition 4.1, to get

$$
c_{1}\left(\mathfrak{s}_{t}\right)^{2}+b_{2}^{-}(W) \leq 4 d_{b}\left(Y_{g,-n}, \mathfrak{t}\right)+4 d\left(S_{-1}^{3}(K)\right)+2 b_{1}\left(Y_{g,-n}\right)+2 b_{1}\left(S_{-1}^{3}(K)\right),
$$

where $\mathfrak{t}=\left.\mathfrak{s}_{t}\right|_{Y_{g,-n}}$. After substituting all the values computed above, this reduces to

$$
\left(-2 \pm 2-\frac{4 g^{2}}{n}\right)+2 \leq 4\left(\frac{1}{4}-\frac{g^{2}}{n}-\frac{n}{4}\right)+4 d\left(S_{-1}^{3}(K)\right)+2(2 g) .
$$

If we take the unfavorable sign on \pm 2 , and recall that $b_{1}(F)=2 g+1$ and $e(F)+1=n$, we get the inequality

$$
\frac{e(F)}{2} \leq 2 d\left(S_{-1}^{3}(K)\right)+b_{1}(F)
$$

This argument relied on a value for $d_{b}\left(Y_{g,-n}\right)$ only valid if $n>2 g$. But the term $d\left(S_{-1}^{3}(K)\right)$ is nonnegative, as can be seen by applying Proposition 4.1 , applied to the surgery cobordism $S^{3} \rightarrow S_{-1}^{3}(K)$. So if $n \leq 2 g$, and consequently $e(F)+2 \leq b_{1}(F)$, then Equation (4.2) still holds.

Remark 4.2. Our final lower bound on the nonorientable four-ball genus is the gap $\frac{\sigma(K)}{2}-d\left(S_{-1}^{3}(K)\right)$. For alternating knots, this quantity is nonpositive — in [10], Ozsváth and Szabó show that

$$
d\left(S_{-1}^{3}(K)\right)=\max \left(0,2\left\lceil\frac{\sigma(K)}{4}\right\rceil\right)
$$

For nonalternating knots, $\frac{\sigma(-)}{2}$ and $d\left(S_{-1}^{3}(-)\right)$ can diverge widely, although both invariants satisfy a crossing-change inequality [12]:

$$
\eta\left(K_{+}\right) \leq \eta\left(K_{-}\right) \leq \eta\left(K_{+}\right)+2 .
$$


If $K$ becomes alternating after $c$ crossing changes, then $\frac{\sigma(K)}{2}-d\left(S_{-1}^{3}(K)\right)$ can be as large as $2 c$.

Remark 4.3. Taking the $-p$-surgery on $K$ instead of -1 -surgery gives a host of other inequalities, one for each of the $p$ distinct $\operatorname{Spin}^{c}$ structures on $S_{-p}^{3}(K)$. These are sometimes stronger: if $F \subset B^{4}$ has boundary $T_{9,5}$, then our inequality certifies that $b_{1}(F) \geq 0$ while an inequality for the -4 -surgery shows that $b_{1}(F) \geq 2$.

\section{Torus knots}

In the section, we apply Theorem 1.3 to torus knots, whose signatures and $d$-invariants are easy to compute. In particular, we will compute the nonorientable slice genus of the family $T_{2 k, 2 k-1}$. Since any surface with boundary $T_{2 k, 2 k-1}$ may be reflected to get a surface with boundary $T_{-2 k, 2 k-1}$, we will focus on the latter (negative) knot.

Signatures of torus knots satisfy a recursion relation (see Theorem 7.5.1 of [8]). If $\sigma(p, q):=\sigma\left(T_{-p, q}\right)$, then

$$
\sigma(p, q)= \begin{cases}\sigma(q, p) & \text { if } q>p \\ \sigma(p-2 q, q)+q^{2}-\epsilon & \text { if } 2 q<p \\ -\sigma(2 q-p, q)+q^{2}-2+\epsilon & \text { if } 2 q>p \\ p-1 & \text { if } q=2 \\ 0 & \text { if } q=1\end{cases}
$$

where $\epsilon=0$ if $q$ is even and $\epsilon=1$ if $q$ is odd.

Let $\sigma_{k}:=\sigma\left(T_{-2 k, 2 k-1}\right)=\sigma(2 k, 2 k-1)$. Applying the first and third conditions twice, we arrive at the recursion

$$
\sigma_{k}=4 k-2+\sigma_{k-1},
$$

whence $\sigma_{k}=2 k^{2}-2$.

The $d$-invariants of torus knots are also simple to compute, since they admit lens space surgeries.

Proposition 5.1 (Proposition 8.1 of [9]). Let $K$ be a knot admitting a positive lens space surgery. Then

$$
d_{-1 / 2}\left(S_{0}^{3}(K)\right)=-\frac{1}{2} \quad \text { and } \quad d_{1 / 2}\left(S_{0}^{3}(K)\right)=\frac{1}{2}-2 t_{0}
$$

where if

$$
\Delta_{K}(T)=a_{0}+\sum_{j=1}^{d} a_{j}\left(T^{j}+T^{-j}\right)
$$

then

$$
t_{0}=\sum_{j=1}^{d} j a_{j} .
$$

The $d$-invariants of zero-surgery are related to those of \pm 1 -surgery via Proposition 4.12 of $[9]$ :

$$
d\left(S_{-1}^{3}(K)\right)=d_{-1 / 2}\left(S_{0}^{3}(K)\right)+\frac{1}{2}, \quad d\left(S_{1}^{3}(K)\right)=d_{1 / 2}\left(S_{0}^{3}(K)\right)-\frac{1}{2} .
$$


Since $T_{p, q}$ admits a positive lens space surgery, we have

$$
d\left(S_{-1}^{3}\left(T_{-p, q}\right)\right)=-d\left(S_{1}^{3}\left(T_{p, q}\right)\right)=-\left(d_{1 / 2}\left(S_{0}^{3}\left(T_{p, q}\right)\right)-\frac{1}{2}\right)=2 t_{0} .
$$

The Alexander polynomial of $T_{p, q}$ is

$$
\Delta_{T_{p, q}}(T)=T^{-(p-1)(q-1) / 2} \frac{(1-T)\left(1-T^{p q}\right)}{\left(1-T^{p}\right)\left(1-T^{q}\right)} .
$$

For torus knots $T_{2 k, 2 k-1}$, the Alexander polynomial has a simple form:

$$
\Delta_{T_{2 k, 2 k-1}}=1+\sum_{j=1}^{k-1} T^{j(2 k-1)}-T^{j(2 k-1)-(k-j)}+T^{-j(2 k-1)}-T^{-j(2 k-1)+(k-j)}
$$

so

$$
t_{0}=\sum_{j=1}^{k-1} j(2 k-1)-\left(j(2 k-1)-(k-j)=\sum_{j=1}^{k-1} k-j=\frac{k^{2}-k}{2} .\right.
$$

Hence

$$
d\left(S_{-1}^{3}\left(T_{-2 k, 2 k-1}\right)\right)=k^{2}-k
$$

The relevant difference between signature and $d$ is

$$
\frac{\sigma}{2}-d=k^{2}-1-\left(k^{2}-k\right)=k-1 .
$$

By Theorem 1.3, we know that

Proposition 5.2. If $F \subset B^{4}$ is a smoothly embedded nonorientable surface with boundary $T_{2 k, 2 k-1} \subset S^{3}$, then $b_{1}(F) \geq k-1$.

To prove Theorem 1.1, apply the proposition to both halves of a surface with cross-section $T_{2 k, 2 k-1}$.

The lower bound in the proposition is tight, as demonstrated by the following general construction. View $T_{p, q}$ as lying in a standard torus, as in Figure 5. Take any two adjacent strands and join them with a strip, or equivalently perform an index 1 Morse move merging them. The resulting cobordism is nonorientable, since the strands were parallel; it is a punctured Möbius band. Since the resulting knot still lives on the torus, it must be $T_{r, s}$ for some $r$ and $s$. The values of $r$ and $s$ can be easily computed by orienting the resulting knot and counting the signed intersection with the horizontal and vertical generators of $H_{1}\left(T^{2}\right)$. A short calculation shows that

$$
r=p-2 t \quad s=q-2 h
$$

where $t \equiv-q^{-1} \bmod p$, with $0 \leq t<p$, and $h \equiv p^{-1} \bmod q$, with $0 \leq h<q$. After an isotopy, $T_{r, s}$ will be in standard, taut form on the torus, and we can repeat the process. Eventually, we arrive at $T_{n, 1}$ for some $n$, which is just an unknot. By concatenating all of these cobordisms, then capping off the final unknot with a disk, we produce a surface $F_{p, q}$ in $B^{4}$ with boundary $T_{p, q}$.

For example, if $p=2 k$ and $q=2 k-1$, we have $t=-(-1)^{-1}=1$ and $h=1^{-1}=1$, giving $r=2 k-2$ and $s=2 k-3$. Thus $T_{2 k, 2 k-1}$ has a $\chi=-1$ cobordism to $T_{2(k-1), 2(k-1)-1}$. Concatenate $k-1$ of these, then cap off $T_{2,1}$ with a disk to get a closed surface $F_{2 k, 2 k-1} \subset B^{4}$ with boundary $T_{2 k, 2 k-1}$ and $b_{1}\left(F_{2 k, 2 k-1}\right)=k-1$. 
Since the isotopies and Morse moves take place inside of the torus, we can actually embed each of these cobordisms in a thickened torus $T^{2} \times[-\epsilon, \epsilon]$ in $S^{3}$, where we view the $[-\epsilon, \epsilon]$ direction as a sort of time. The obstruction to embedding all of $F_{p, q}$ in $S^{3}$ is that the final disk with boundary $T_{n, 1}$ cuts through all of the previous layers unless $n=0$. To get a surface in $S^{3}$, we must continue with these within-torus cobordisms: $T_{n, 1} \mapsto T_{n-2,1} \mapsto \cdots$. If $n$ is even, or equivalently if $p q$ was even to start, then we do get a surface in $S^{3}$. Teragaito has shown that these surfaces achieve the minimal $b_{1}$ among nonorientable surfaces in $S^{3}$ with boundary $T_{p, q}$ [15]. If $n$ is odd, then this construction fails to give a surface in $S^{3}$, though a slight modification (cf. [15] Remark 4.9) will do.

The first Betti numbers of the $F_{p, q}$ do not have a simple closed form: they obey the recursion $b_{1}\left(F_{p, q}\right)=b_{1}\left(F_{p-2 t, q-2 h}\right)+1$ where $t$ and $h$ are the minimal nonnegative representatives of $-q^{-1}$ modulo $p$ and $p^{-1}$ modulo $q$, respectively.

We conjecture that the surfaces $F_{p, q}$ have minimal $b_{1}$ among all smoothly embedded surfaces in $\mathbb{R}^{4}$ with boundary $T_{p, q}$. Many pairs $(p, q)$ for which this conjecture holds can be certified using the $d$-invariant bounds of this paper. Similar invariants, derived by considering larger surgeries on the knot, give even more examples. The smallest knot for which the conjecture remains open is $T_{7,4}$, which is known to be the boundary of a punctured Klein bottle but may yet be the boundary of a Möbius band.

\section{Acknowledgments}

The author would like to thank Peter Ozsváth for many helpful conversations, and Jesse Wolfson, Jacob Rasmussen and Charles Livingston for comments on a draft. Thanks to the referees for careful reading and comments. This material is based upon work supported by the National Science Foundation Graduate Research Fellowship under grant no. DGE-1122374.

\section{References}

[1] R. Fox and J. Milnor, Singularities of 2-spheres in 4-space and cobordism of knots, Osaka J. Math. 3 (1966) 257-267.

[2] I.M. Gessel, A.S. Levine, D. Ruberman and S. Strle, Non-orientable surfaces in homology cobordisms, 2013, arXiv:1310.8516.

[3] P. Gilmer and C. Livingston, The nonorientable 4-genus of knots, J. London Math. Soc. 84(3) (2011), 559-577.

[4] C. Gordon and R. Litherland, On the signature of a link, Invent. Math. 47(1) (1978), 53-69.

[5] P. Kronheimer and T. Mrowka, Gauge theory for embedded surfaces. I, Topology 32(4) (1993), $773-826$.

[6] H. Murakami and A. Yasuhara, Four-genus and four-dimensional clasp number of a knot, Proc. Amer. Math. Soc. 128(12) (2000), 3693-3700.

[7] K. Murasugi, On a certain numerical invariant of link types, Trans. Amer. Math. Soc 117 (1965), 387-422.

[8] — Knot theory \& its applications, Modern Birkhäuser Classics, Birkhäuser Boston Inc., Boston, MA (2008), ISBN 978-0-8176-4718-6. Translated from the 1993 Japanese original by Bohdan Kurpita.

[9] P. Ozsváth and Z. Szabó, Absolutely graded Floer homologies and intersection forms for fourmanifolds with boundary, Adv. Math. 173(2) (2003), 179-261.

[10] - Heegaard Floer homology and alternating knots, Geom. Topol. 7(1) (2003), 225-254.

[11] —, Knot Floer homology and the four-ball genus, Geom. Topol. 7(2) (2003), 615-639.

[12] T. Peters, A concordance invariant from the Floer homology of \pm 1 surgeries, 2010, arXiv: 1003.3038. 
[13] J. Rasmussen, Khovanov homology and the slice genus, Invent. Math. 182(2) (2010), 419-447.

[14] S. Suzuki, Local knots of 2-spheres in 4-manifolds, Proc. Japan Acad. Ser. A, Math. Sci. 45(1) (1969), 34-38.

[15] M. Teragaito, Crosscap numbers of torus knots, Topol. Appl. 138(1-3) (2004), 219-238.

[16] A. Yasuhara, Connecting lemmas and representing homology classes of simply connected 4manifolds, Tokyo J. Math. 19(1) (1996), 245-261.

Department of Mathematics, Massachusetts Institute of Technology, 77 Massachusetts Avenue, Cambridge, MA, USA

E-mail address: joshuab@alum.mit.edu 